\title{
GEOKINEMATICS IN GGOS - DIFFERENT RELATIONS AND DEPENDENCES
}

\author{
Wojciech Pachelski ${ }^{(1)}$, Małgorzata Paśnicka ${ }^{(2)}$, Karolina Szafranek ${ }^{(1)}$, \\ Agnieszka Zwirowicz-Rutkowska ${ }^{(3)}$ \\ (1) Military University of Technology, Warsaw, POLAND \\ (2) Space Research Centre of PAS, Warsaw, POLAND \\ (3) University of Warmia and Mazury, Olsztyn, POLAND
}

\begin{abstract}
The paper presents the preliminary results of the analysis of fundamental dependencies between different geodetic concepts related to the one of the GGOS (Global Geodetic Observation System) pillars called Geometry and Kinematics. These relationships are described using UML (Unified Modelling Language) - one of the graphical notations that can be used for information modelling. Concepts are represented as classes with their names, attributes, and different kinds of links between them. The main purpose of this paper is to introduce the full description of connections between all GGOS components.
\end{abstract}

Keywords: GGOS, kinematics, measurement techniques

\section{INTRODUCTION}

Geodesy is the science of determining the geometry, gravity field, and rotation of the Earth. It also describes their evolution in time. Recently, especially due to satellite techniques and accuracy improvement, geodesy plays a significant role in studying the Earth system. The description of this system is very difficult because of continuous changes (e.g. climate change), inherent dynamics and many complex relations between its components. Data collected using various techniques complement one another. GGOS stands for Global Geodetic Observing System and it was established by the International Association of Geodesy (IAG) in July 2003. The idea was to combine all geodetic measurement techniques to take full advantage of them. GGOS is a part of the Global Earth Observation System of Systems (GEOSS). The project is important because it allows a more complete understanding of the Earth's characteristics. Observations and other data products are provided not only to scientists but also for non-scientific applications (IAG services). Observations serve also as the basis for many societal areas: disaster prevention, provision of resources like energy and water, protection of the biosphere, and many others (Plag et al., 2009).

The main purpose of this paper is to introduce the full description of complex connections between GGOS components. Understanding the relations and dependences within GGOS is necessary for appropriate usage of its products. The authors' intention is to show and explain examples of such relations referred to the part of GGOS that is described as 'Geokinematics' and to present these relations using the Unified Modelling Language (UML) structure diagrams (Fig. 1). 
UML is the formal language that is used to model and describe reality in object-oriented analysis and programming (OMG UML, 2010). Analysis of interrelations between observation techniques and the Earth's gravity field were presented in Pachelski et al. (2008).

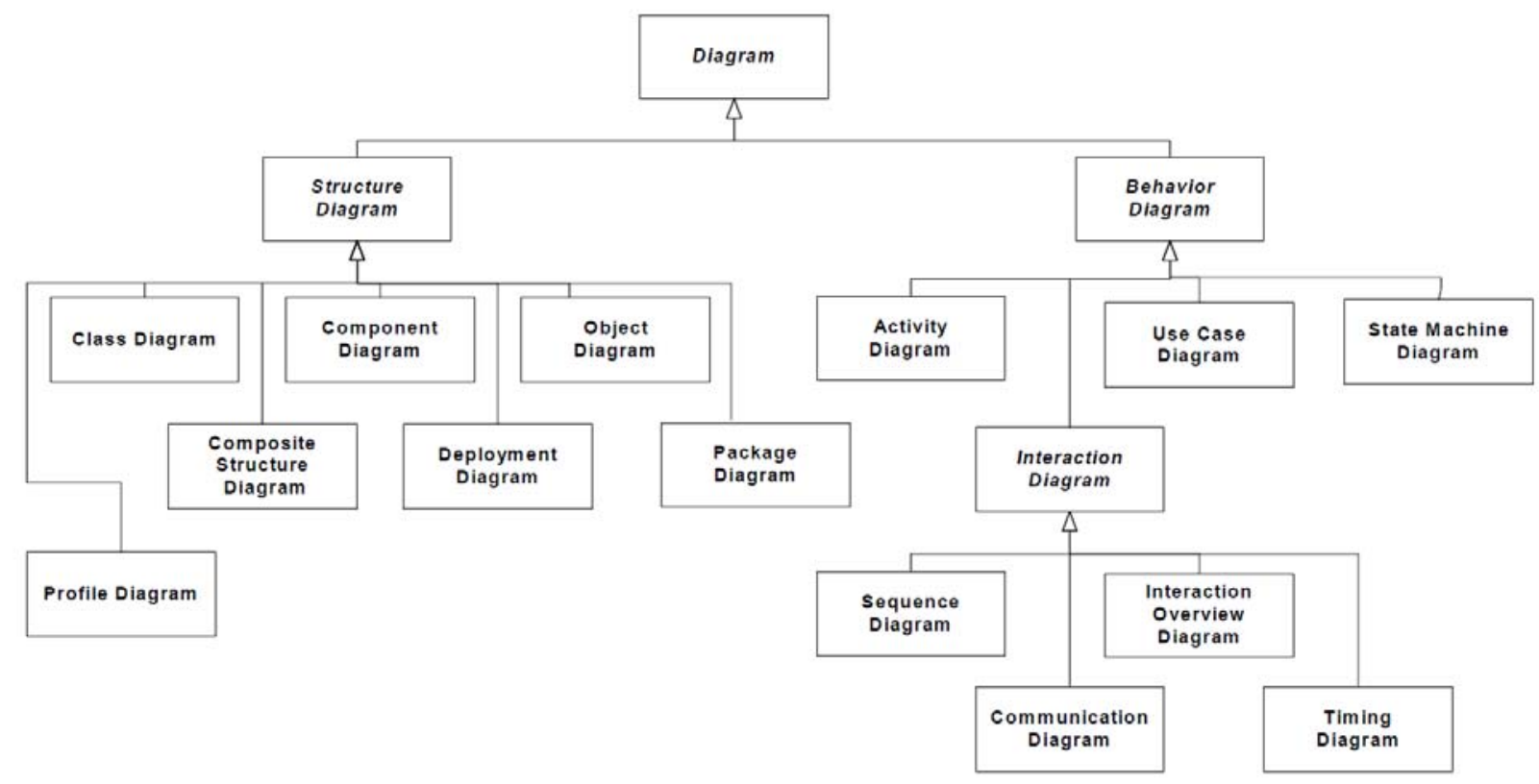

Fig. 1. The structure of UML diagrams

\section{GGOS STRUCTURE}

GGOS divides geodesy as a discipline into three parts, the so called "pillars". The first pillar consists of methods, techniques and theories that are used to determine the Earth's shape (its surface: the solid Earth, ice and oceans) as a global function of space and time ("Geokinematics"). The second pillar relates to the Earth's gravitational field determination and monitoring and also describes mass distributions and the shape of the geoid. The third pillar concerns the planet rotation and forces related to interactions between the Earth and other celestial bodies, especially the Moon and the Sun. These three pillars constitute the province of modern geodesy (Fig. 2, Plag and Pearlman, 2009). Figure 3 presents different UML packages of classes assigned to four fundamental pillars that represent the GGOS structure. Each of the packages is to be developed into an UML class diagram.

The purpose of this paper is to develop the package "Geokinematics" and its relationships to the "GravityField"1 and "ReferenceFrame" packages.

\footnotetext{
${ }^{1}$ UML names of packages, classes, attributes and other constructs are built up according to the conventions adopted in the ISO 19100 series of standards (ISO, 2005).
} 


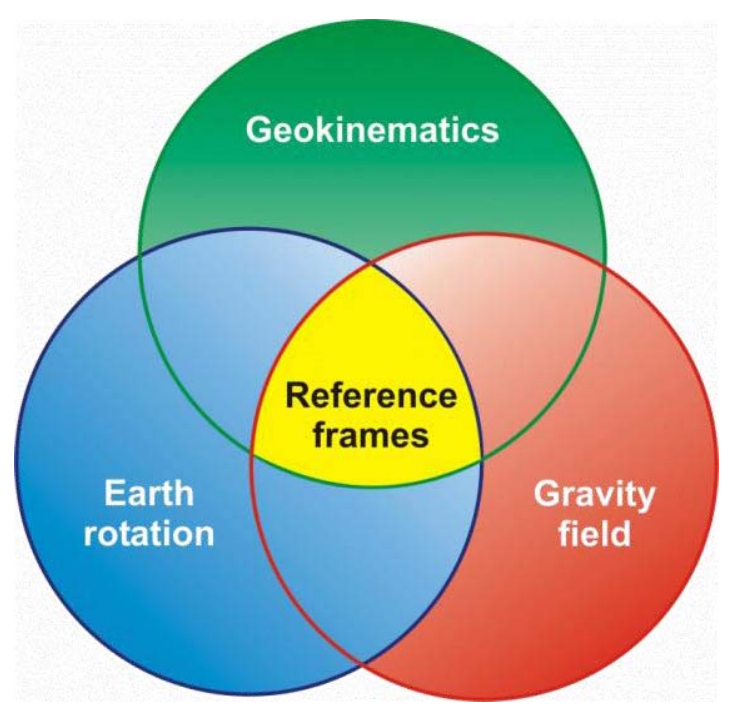

Fig. 2. Three permeated pillars with a special role of Reference frames, (Plag and Pearlman, 2009)

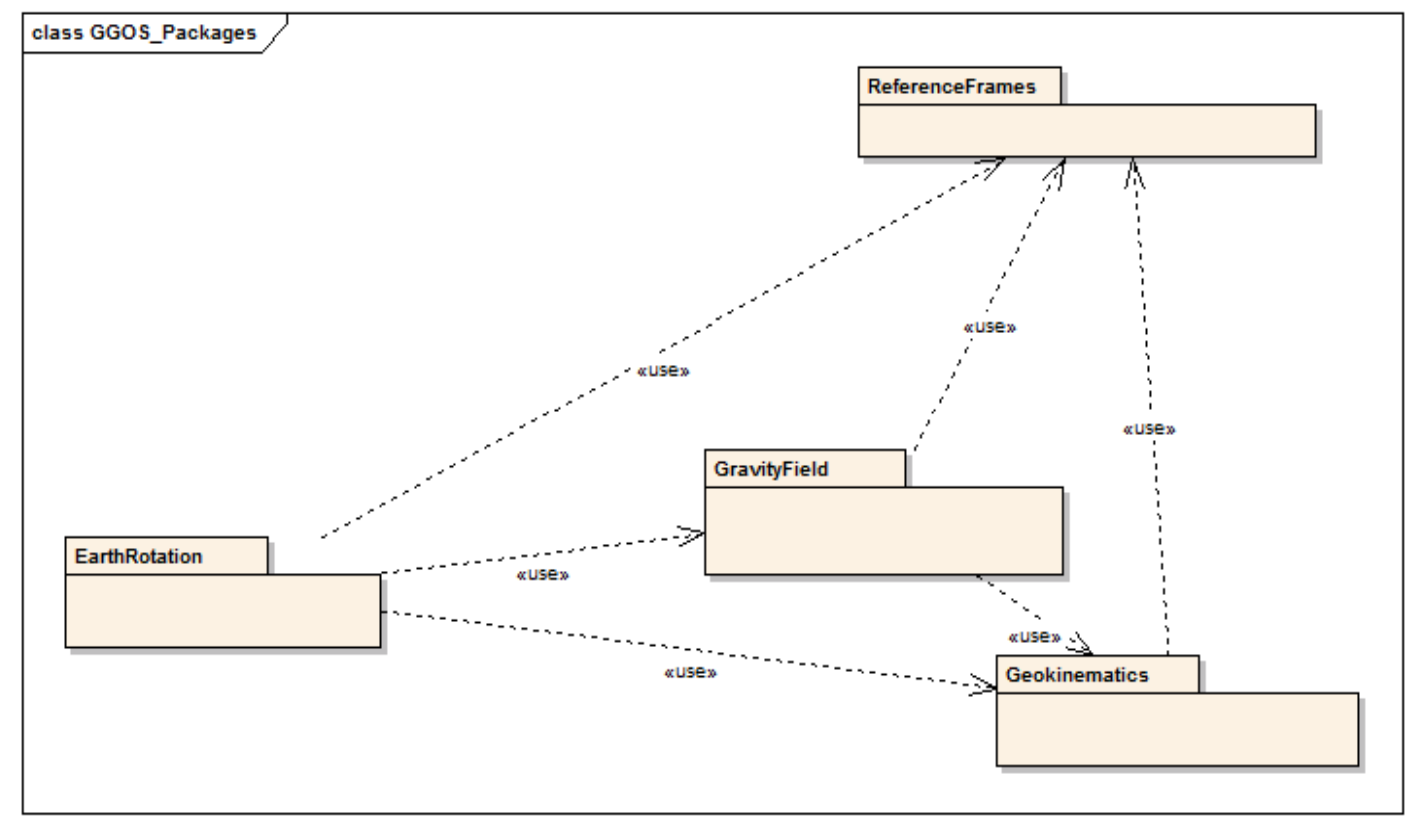

Fig. 3. Three GGOS pillars presented as an UML package diagram

There are also several connections and dependences between the pillar components. The section containing reference frames plays a special role here since it joins all three other pillars together and it contains data that are necessary to properly situate results of the measurements (Fig. 2). Assigning different techniques to proper pillars can be only approximate since data products obtained using one technique can be used for many different applications, such as GNSS (Fig. 4). In this paper the authors analyze measurement techniques assigned especially to kinematics and show their role in definition and maintenance of the reference frame. The relations to other pillar components are also taken into account. The first step of these studies is answering the questions: what kind of value is directly measured by the analyzed technique and what is the final product of this technique. It is given here only a brief discussion of such studies for selected techniques that are related mainly to the Geokinematics (Table 1). 


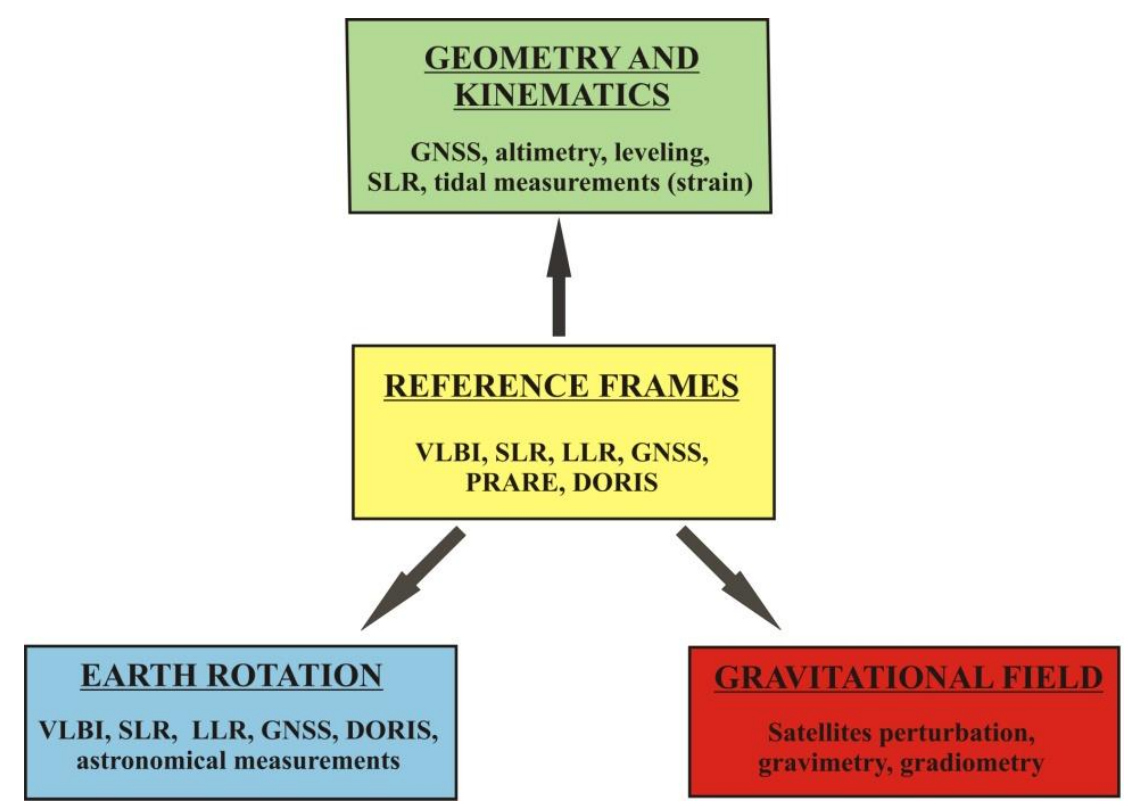

Fig. 4. Different measurement techniques assigned to GGOS pillars

Table 1. Measurement techniques related to geometry and kinematics

\begin{tabular}{|c|l|l|l|}
\hline Technique & \multicolumn{1}{|c|}{ Measured values } & \multicolumn{1}{|c|}{ Final products } & \multicolumn{1}{c|}{ GGOS pillar } \\
\hline GNSS & $\begin{array}{l}\text { Distances between receiver } \\
\text { and satellite (phase or code } \\
\text { method), time; distance } \\
\text { differences }\end{array}$ & $\begin{array}{l}\text { Ellipsoidal coordinates of antenna } \\
\text { phase centre, site velocities; } \\
\text { absolute or relative ground point } \\
\text { positions. }\end{array}$ & $\begin{array}{l}\text { Geokinematics, } \\
\text { Reference frames }\end{array}$ \\
\hline Altimetry & $\begin{array}{l}\text { Distances between satellite } \\
\text { and instantaneous sea surface }\end{array}$ & Geopotential models, geoid's shape & $\begin{array}{l}\text { Geokinematics, } \\
\text { Gravitational field }\end{array}$ \\
\hline $\begin{array}{c}\text { Precise } \\
\text { leveling }\end{array}$ & Height differences, gravity & $\begin{array}{l}\text { Heights or height differences } \\
\text { (expressed in a given height system) }\end{array}$ & $\begin{array}{l}\text { Geokinematics, } \\
\text { Gravitational field }\end{array}$ \\
\hline SLR & Satellite-to-station distances & $\begin{array}{l}\text { Position of geocentre, station } \\
\text { positions and velocities in a given } \\
\text { reference frame }\end{array}$ & $\begin{array}{l}\text { Reference frames, } \\
\text { Earth rotation } \\
\text { (orientation) } \\
\text { Geokinematics }\end{array}$ \\
\hline
\end{tabular}

\section{INTRODUCTION TO GEOMETRY AND KINEMATICS}

Geometry and kinematics refer to the determination of object positions and their change in time (movement). Defining a suitable reference system (and its realization as a reference frame) is crucial. This part of geodesy can be divided into two main parts. The first is referred to the Earth's shape and size determination and the second to determine the object positions (especially geodetic points) in the selected reference frame using orthocartesian (X, Y, Z) or geodetic (North, East, Up) coordinates.

The shape of the Earth is usually approximated using a reference ellipsoid of revolution with two semi-axes (Fig. 5). As an example we have the Geodetic Reference System 1980 (GRS'80), which was adopted in 1979 in Canberra during the International Union of Geodesy and Geophysics (IUGG) XVII General Assembly. GRS'80 represents the size, shape and gravity field. The system is based on a geocentric, equipotential ellipsoid that is defined using four standard values of the Earth: its equatorial radius $a$, geocentric gravitation constant $G M$, Earth's dynamic shape coefficient $J_{2}$ and angular velocity of the planet $\omega$ (Czarnecki, 1997). These parameters are shown as attributes of the classes 'ReferenceEllipsoid' and 'GeocentricEllipsoid' in Fig. 5. These values are sufficient to determine all other dynamic and geometric parameters of the reference ellipsoid because, due to 
Stokes' theory, size, shape, mass and angular velocity define unambiguously the function describing the normal potential (potential generated by an ellipsoid). The definition of the system takes into consideration all three GGOS pillars (Earth's geometry, rotation and potential), but here we want to emphasize its geometric part (shape and size of the planet) as the base for the analyzed pillar. Due to the simple mathematic description of an ellipsoid's shape, it is a very good approximation of the Earth and is used to characterize the geoid figure and its undulations (its heights above ellipsoid, Fig. 5). The shape of geoid is too complex and irregular to be described by means of a mathematic formula.

Nowadays the World Geodetic System 84 (WGS'84) is a standard reference system for use in geodesy, cartography and navigation, but its geometrical parameters are almost the same as GRS' 80 , so the IAG Sub-committee for European Reference Frame (EUREF, http://www.euref-iag.net/) decided to use GRS' 80 for the European Terrestrial Reference System (ETRS89).

The second task of Geokinematics is the determination of object positions in space and their changes in time. Point positions can be expressed in many different reference frames, so usage of a proper one is very important. Usually positions can be expressed using geocentric orthocartesian (X, Y, Z) or geodetic (North, East, Up) coordinates (Fig. 5). Determination of geodetic coordinates (especially in global, regional and national networks) has to be done with the highest possible accuracy and precision (e.g. permanent GNSS sites and precise processing of data), but the position for navigation can be less accurate (e.g. single-frequency GPS receivers). Coordinates obtained using GNSS are typically geometric as heights of points above the reference ellipsoid (along the normal line). In geodesy, however, heights are defined using the difference between the geoid's potential and the potential of the equipotential surface containing measured point, so they are strictly related to the Earth's potential (another pillar). It is impossible to determine altitudes without taking into consideration the gravitational field of the planet. The most fundamental relationships between elements of the GGOS pillars (Geokinematics in relation to others, especially Gravity Field and Reference Frames) are shown in Figure 5.

\section{HEIGHT SYSTEMS AS AN EXAMPLE OF RELATIONS BETWEEN GGOS PILLARS (GEOKINEMATICS AND GRAVITY FIELD)}

There are many different systems for describing heights. The fundamental (geodetic) definition of height is strictly connected with the Earth's gravity. All systems are based on a value called the geopotential number C (Fig. 5) that expresses the difference between the geoid's potential and the potential of a surface, which contains a given point R:

$$
C_{R}=W_{0}-W_{R}=\int_{0}^{R} g \cdot d h
$$

This expression identifies the work that is needed to counteract gravity during the movement of the unit mass from the point $O$ on the geoid (with potential $W_{0}$ ) to the point where the potential is equal to $W_{R}\left(W_{R}<W_{0}\right)$. This work does not depend on the distance between two points and this is an attribute that is different from the common understanding of height (and from geometric heights). To obtain heights - the distance along the gravity field $W$ gradient between the two surfaces - the geopotential number has to be divided by a force (acceleration for a unit mass) that is representative of the distance between the geoid and a point $\mathrm{R}$ along the plumb line. This procedure shows clearly the inseparable relation between the Earth's gravity field and the position determination. The method of a representative acceleration determination specifies the system of height measurement in geodesy (Czarnecki, 1997). 
For geopotential heights, the constant value of $10 \mathrm{~m} / \mathrm{s}^{2}$ is set for dynamic systems - the constant value for normal acceleration of gravity at latitude $45^{\circ}$ (calculated e.g. using the Helmert formula). The determination of accelerations for orthometric and normal systems is more complicated. For orthometric heights an average value of the acceleration of gravity $g$ is needed. To obtain it, the acceleration $g$ on the Earth's surface has to be measured. The second step is to reduce this value to get an acceleration corresponding to the point in the middle of the $R-O$ section. The disadvantage of this method is that spatial mass distribution along the plumb line has to be known to use Poincaré-Prey reduction. The solution of this problem is to take advantage of Molodensky's theory and the system of the normal heights that provides a value called the mean normal acceleration $\gamma$ of gravity between the points $R$ and $O$. This is easy to calculate using the Helmert formula for obtaining normal acceleration on the ellipsoid surface as a function of latitude and the inverted free-air reduction to get a normal acceleration corresponding to the point in the middle of the $R-O$ section. These values of proper acceleration are attributes of different Height System classes (Fig. 5). This short description of height systems emphasizes a very strong dependence between position determination and the Earth's gravity field. Due to the correlation of gravity with the Earth's rotation, as gravity is the result of the Earth's mass and centrifugal force, height is the term that links together all three GGOS pillars.

Nowadays, satellite systems play a significant role in geodesy and navigation. Determination of coordinates using satellite systems (e.g. GPS-NAVSTAR: Global Positioning System NAVigation Signal Timing and Ranging) is geometrical. Coordinates are originally expressed in an Earth-centered Earth-fixed (ECEF) frame consistent with the International Terrestrial Reference Frame (ITRF). After their transformation to the European Terrestrial Reference System (ETRF) as North, East, Up instead of X, Y, Z they are still strictly geometrical, so height (an Up component) obtained using GNSS does not contain any information about work that should be done to move a unit mass. It is not related to the Earth's gravitational field. Geometrical heights are defined as the parts of geometric plumb lines (or normal lines to the ellipsoid due to the small curvature of plumb lines) measured from the surface of the geocentric ellipsoid GRS'80/WGS'84 to the ellipsoid that contains the point from which height is being determined. The distance from the surface of the ellipsoid (not necessarily geocentric) measured along normal lines is called the 'geodetic' height. The terms 'geodetic' and 'geometric' are often used alternatively. Geometric heights constitute a separate class on Fig. 5.

With the increasing use of satellite methods and due to normal and orthometric system usage in Europe, satellite levelling becomes an important part of geodetic measurements (Czarnecki, 1997). Using geopotential models (determined by means of different satellite techniques) or plumb line declination, the geoid undulations can be derived (Fig. 5). They join the ellipsoid as a geometrical surface (Geokinematics pillar) and the geoid - surface of the constant potential value (Gravity Field pillar). It is important that both ellipsoid and geoid constitute a reference for different types of measurements and they approximate the Earth's shape. Satellite levelling uses undulations to determine orthometric heights based on measured geometric height values.

\section{NORMAL AND REAL EARTH POTENTIAL AS AN EXAMPLE OF RELATIONS BEETWEN GGOS PILLARS}

Since gravity on the Earth's surface is the result of both the gravitational force and centrifugal force related to rotation, it accounts for an unbreakable connection between the Earth Rotation and the Gravity Field GGOS pillars.

As an example of many mutual relations between pillar components GRS'80 (Global Reference System 1980) can be given (Czarnecki, 1997). This system is based on the geocentric 
equipotential ellipsoid defined using four standard values of the Earth: equatorial radius $a$, geocentric, gravitational constant $G M$, angular velocity $\omega$ and dynamical factor of shape $J_{2}$, which describe normal potential on the Earth's surface and determine all other geometric and dynamical values. All of them are attributes of classes called 'GeocentricEllipsoid' and 'ReferenceEllipsoid' in Fig. 5. The Clairaut theorem characterizes the relations between all GGOS pillar elements: $f-$ Earth's geometrical flattening (Geokinematics), $f^{*}$ - Earth's geodynamical flattening (GravityField) and $q$ - ratio of the acceleration caused by centrifugal force (on the equator) to gravitational acceleration in the same point (EarthRotation and GravityField).

Equation (1) enables geometrical flattening determination using only geodynamical values, so it joins all three GGOS segments' elements:

$$
f+f^{*}=\frac{5}{2} q
$$

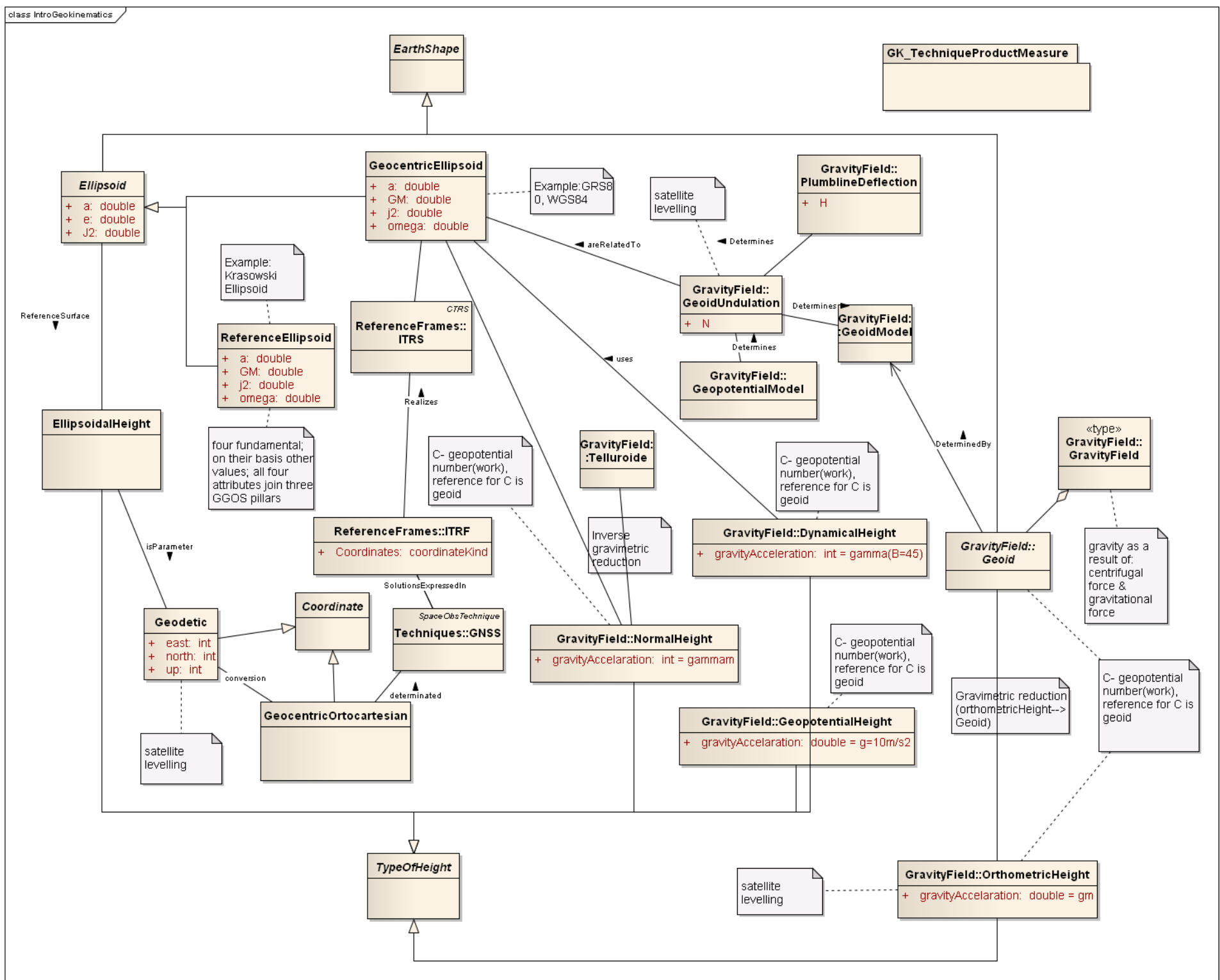

Fig. 5. Fundamental relationships between elements of GGOS pillars (Geokinematics in relation to Gravity Field and Reference Frames) 


\section{GNSS AS AN EXAMPLE OF SATELLITE SYSTEMS AND THEIR ROLE IN GEOKINEMATICS}

Nowadays, satellite systems play a significant role in geodesy. They help to describe the Earth's shape (altimetry missions e.g. Jason, TOPEX/Poseidon) and the Earth's gravity field (e.g. GOCE, GRACE). GNSS (Global Navigation Satellite Systems) are particularly important for the Geokinematics GGOS pillar. Currently the two systems are operational: American GPS-NAVSTAR and Russian GLONASS (ГЛОНАСС, Глобальная навигационная спутниковая система; Global Navigation Satellite System ). European Galileo and Chinese COMPASS are under development. All of them are shown in Fig. 6. They enable position determination and reference frames e.g. ITRF (International Terrestrial Reference Frame) and ETRF (European Terrestrial Reference Frame) creation and maintenance. Long-term observations provide data for geophysical and geological research, especially velocities and vertical movement determination (Blewitt and Lavallée, 2002).

GPS uses WGS84 as an approximation of the Earth's shape and the reference for North, East, and Up components (Geokinematics pillar), originally obtained in orthocartesian coordinates. Satellite ephemerides are expressed in an ECEF frame consistent with the ITRF (which rotates with the Earth), the Earth rotation parameters are taken into consideration during data processing (the Earth Rotation pillar), and some of them can be determined from advanced data processing.

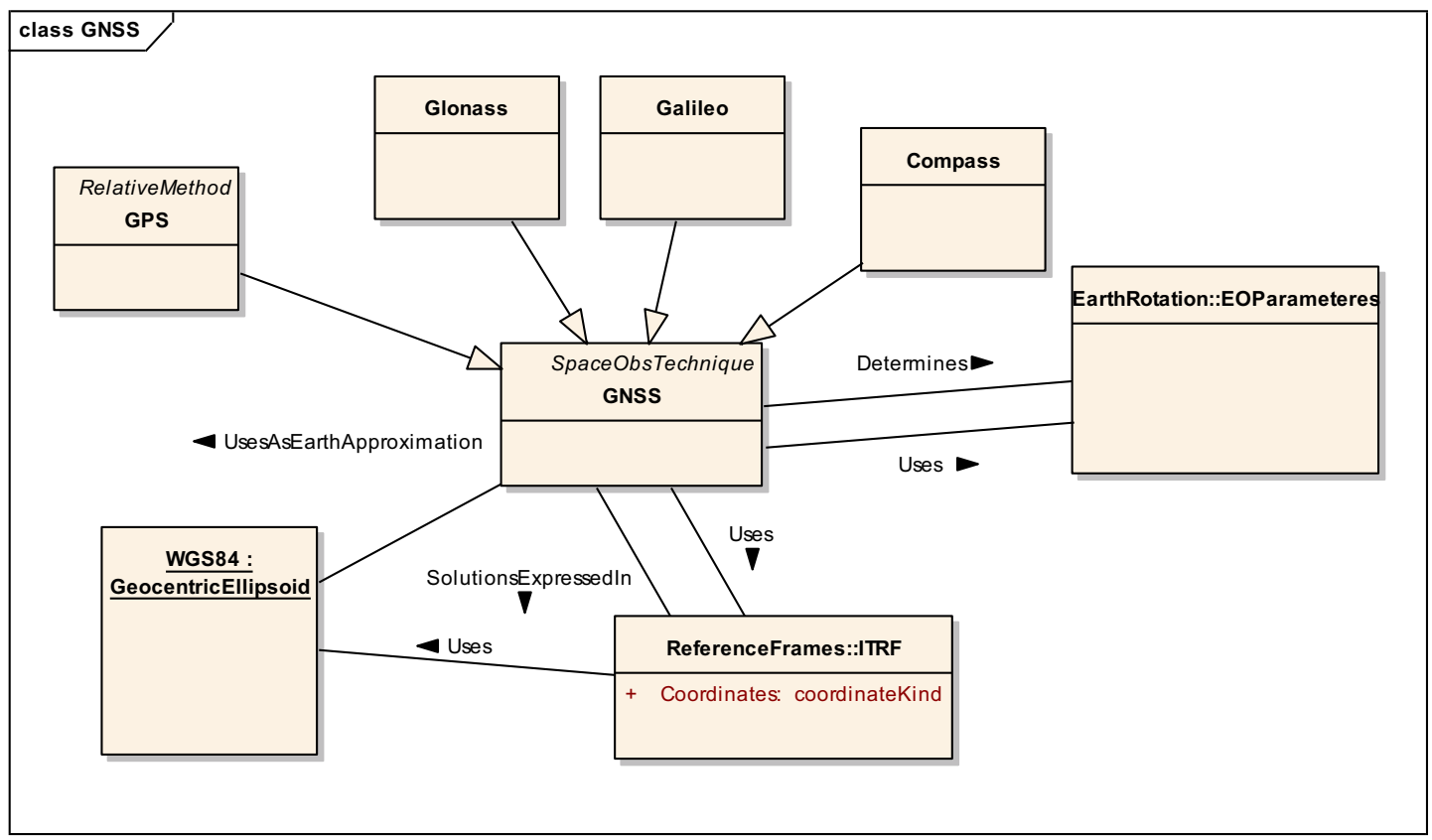

Fig. 6. Diagram of fundamental relationships concerning GNSS class

Satellites are held in orbit by gravitational force (Gravity Field pillar). During data processing many models have to be implemented e.g. tidal model describing changes of the Earth's shape that are the result of gravitational interaction between the Earth, the Moon and the Sun. The scheme describing this procedure is more complex due to 'feedback' - e.g. the troposphere model has to be implemented for precise determinations, but it is also an outcome of this process. As a result of the processing of permanent networks, e.g. IGS - International GNSS Service (http://igscb.jpl.nasa.gov/), EPN - European Permanent Network (http://www.epncb.oma.be/) and national networks, precise coordinates and their changes in time - velocities are obtained (Geokinematics pillar). They are used for reference system realization (Reference Frames e.g. ITRF, ETRF). A detailed description of satellite data calculation will be presented during basic diagram development in another paper. 


\section{SUMMARY}

This paper presents a description of the main relationships between different classes of the Geokinematics GGOS pillar and classes of another GGOS segments, especially the Gravity Field and Reference Frames. The primary goal was to show a very complex structure of dependences between different segments and to describe geodesy using standardized general-purpose modelling language UML. It is impossible to analyse one selected segment of geodesy individually without accounting for connections with others. The Reference Frames have a crucial role here because they are necessary for all geodetic measurements. All diagrams presented are the result of preliminary analysis - they play a major role of the frame for further, more detailed analysis. Modelling of all the most important GGOS relations will facilitate ordering all terms and elements and joining different parts of geodesy.

\section{ACKNOWLEDGEMENT}

The research was supported by the grant No. N N526 159836 („Core information models of the main theoretical, methodological and observational segments of the Global Geodetic Observing system GGOS") of the Polish Ministry of Science and Higher Education.

The authors would like to express their gratitude to the Reviewer for many helpful comments.

\section{REFERENCES}

Blewitt G., Lavallée D. (2002): Effect of annual signals on geodetic velocity, J. Geophys. Res., 107(B7), 2145, doi:10.1029/2001JB000570.

Czarnecki K. (1997): Geodezja współczesna w zarysie, Wiedza i Życie, ISBN: 83-86805-67-6

European Permanent Network: http://www.epncb.oma.be/

International Association of Geodesy, On Geodesy and the Global Geodetic Observing System: http://www.iag-ggos.org/

International Association of Geodesy, Reference Frame Sub Commission for Europe: http://www.euref-iag.net/

International Earth Rotation and Reference Systems Service: http://www.iers.org

International GNSS Service: http://igscb.jpl.nasa.gov/

ISO (2005): ISO/TS 19103 Geographic information - Conceptual schema language.

OMG (2010): Unified Modeling Language TM (OMG UML) Superstructure, Version 2.3 (2010), http://www.omg.org/spec/UML/2.3/Superstructure/PDF/

Pachelski W., Paśnicka-Pawłowska M., Szafranek K., Zwirowicz A. (2008): GGOS information models: analysis of interrelations between observation techniques and the Earth gravity field Reports on Geodesy No. 2 (85), pp. 61-70

Plag, H.-P. \& Pearlman, M., eds. (2009): Global Geodetic Observing System: Meeting the Requirements of a Global Society on a Changing Planet in 2020, Springer Berlin, ISBN: 978-3642-02686-7, DOI: 10.1007/978-3-642-02687-4_1-11.

Plag, H.-P., Gross, R., Rothacher, M. (2009): Global Geodetic Observing System for Geohazards and Global Change, Geosciences, BRGM's journal for a sustainable Earth, 9, pp. 96-103

Received: 2011-05-10,

Reviewed: 2011-06-13, by B. Luzum,

Accepted: 2011-07-26. 